\title{
Development and Validation for Reverse Phase High Performance Liquid Chromatographic Method for Simultaneously Determination for Diphenoxylic Acid, Nitrileamide and Bromdiphenyl Butoro Nitrile in Diphenoxylate Hydrochloride Bulk Drug
}

\author{
R. S. Lokhande ${ }^{1}$, P. U. Singare ${ }^{2, *}$, P. V. Jadhav ${ }^{1}$ \\ ${ }^{1}$ Department of Chemistry, University of Mumbai, Vidyanagari, Santacruz, 400098, Mumbai \\ ${ }^{2}$ Department of Chemistry, Bhavan's College, Andheri, 400058, Mumbai \\ pravinsingare@gmail.com
}

\begin{abstract}
Related substances by HPLC method was developed and validated for Diphenoxylate hydrochloride bulk drug and its three potential impurities i.e. Diphenoxylic acid (degradation product), Nitrile amide (precursor) and Bromo Diphenyl butro nitrile (precursor). Analysis was performed on AGILENT 1100 SERIES HPLC system with auto injector and binary gradient high pressure mixing pump on Inersil ODS-3 (RP C 18) column. Mobile phase of Acetonitrile and HPLC water pH adjusted to 2.3 with orthophosphoric acid in the ratio of $25: 75(\mathrm{v} / \mathrm{v})$ with change in the ratio of mobile phase by linear gradient to $85: 25(\mathrm{v} / \mathrm{v})$ in 45 minute was pumped at $2 \mathrm{~mL} / \mathrm{min}$ and the detection was done at $210 \mathrm{~nm}$. The parameter for which the method was validated included specificity, limit of detection and quantitation, linearity, precision, accuracy and robustness. The method was successfully used to quantitate the levels known impurities \{i.e. Diphenoxylic acid (degradation product), Nitrileamide (precursor) and Bromo Diphenyl butro nitrile (precursor) $\}$ and unkown impurities in Diphenoxylate hydrochloride bulk drug.
\end{abstract}

Keywords Diphenoxylate Hydrochloride, HPLC, Reverse Phase HPLC, Validation, Quantification, Impurity Profiling

\section{Introduction}

Official method for bulk drug analysis of Diphenoxylate hydrochloride is carried out by thin layer chromatography on reverse phase plate ${ }^{1-2}$ and the impurities were compared with diluted standard. Literature ${ }^{3-8}$ reveals several liquid chromatographic methods for determination of Diphenoxylate hydrochloride but not much interest has been taken towards the generation of impurity profile of the drug. In this contest the present work reports a reverse phase HPLC Method for separation and estimation of Diphenoxylate hydrochloride and its known impurities (i.e. Diphenoxylic acid, Nitrileamide and Bromo diphenyl nitrile) and unknown impurities by using their relative retention time and Relative retention factor.

\section{Experimental}

* Corresponding author:

pravinsingare@gmail.com (P.U. Singare)

Published online at http://journal.sapub.org/fs

Copyright (C) 2012 Scientific \& Academic Publishing. All Rights Reserved

\subsection{Chemical and Reagents}

Working standard of Diphenoxylate Hydrochloride, Working standard of Bromo Diphenyl Butyro Nitrile, Working standard of Nitrileamide and Working standard of Diphenoxylic acid were obtained by RPG Life Sciences Ltd with certificate of analysis. Water and Acetonitrile used were HPLC grade obtained from E. Merck India Ltd.

\subsection{Preparation of Standard Solution (Working Concentration Level)}

Individual stock solution of Diphenoxylate hydrochloride and its three solutes containing $5 \mathrm{ug} / \mathrm{mL}$ (i.e. $0.5 \%$ with respect to concentration of Diphenoxylate Hydrochloride) were prepared by diluting with diluent. The initial solution was $100 \mathrm{ug} / \mathrm{mL}$ prepared in diluent. Mixture of requisite compositions was obtained by mixing appropriate aliquots of the four solutions (System suitability solution at working concentration level).

\subsection{Preparation of Test Solution}

The test solution of $1000 \mathrm{ug} / \mathrm{mL}$ was prepared in diluent. 
R. S. Lokhande et al.: Development and Validation for Reverse Phase High Performance Liquid Chromatographic

Method for Simultaneously Determination for Diphenoxylic Acid, Nitrileamide

and Bromdiphenyl Butoro Nitrile in Diphenoxylate Hydrochloride Bulk Drug

\subsection{HPLC Instrumentation}

Agilent 1100 series HPLC with high pressure gradient binary pump, UV detector, Auto sampler and Chemstation software. The analyte peaks were resolved on Inertsil ODS 3, $250 \times 4.6 \mathrm{~mm}$. 5 micron HPLC analytical column. The chromatographic conditions are listed below;

\section{HPLC chromatography condition}

i. Flow rate: $2.0 \mathrm{~mL} / \mathrm{min}$

ii. Injection volume: $20 \mu \mathrm{L}$

iii. Detector: UV $210 \mathrm{~nm}$

iv. Solution A: Water $\mathrm{pH}$ adjusted to 2.3 with orthophosphoric acid

v. Solution B: Acetonitrile

vi. Diluent: Solution A: Solution B : 50:50

vii. Mobile phase: Binary gradient as described below

\begin{tabular}{|c|c|c|c|}
\hline TIME & SOLUTION A & SOLUTION B & FLOW RATE (mL/min) \\
\hline 0 & 75 & 25 & 2.0 \\
\hline 5 & 75 & 25 & 2.0 \\
\hline 40 & 15 & 85 & 2.0 \\
\hline 45 & 75 & 25 & 2.0 \\
\hline
\end{tabular}

Table 1. Relative Retention time and Response factor of Diphenoxylate hydrochloride and its impurities

\begin{tabular}{|c|c|c|c|}
\hline No. & Identity & $\begin{array}{c}\text { Relative } \\
\text { Retention } \\
\text { time } \\
\text { (RRT) }\end{array}$ & $\begin{array}{c}\text { Relative } \\
\text { Response } \\
\text { factor } \\
\text { (RRF) }\end{array}$ \\
\hline 1 & Diphenoxylic acid & 0.78 & 1.27 \\
\hline 2 & Diphenoxylate Hydrochloride & 1.00 & 1.00 \\
\hline 3 & Nitrile amide & 1.85 & 0.96 \\
\hline 4 & Bromo diphenyl Butro nitrile & 2.10 & 1.23 \\
\hline
\end{tabular}

Note: Relative retention time and relative response factor were calculated with respect to retention time and peak response of Diphenoxylate hydrochloride.

Table 2. Typical Retention Time of Diphenoxylate hydrochloride and its impurities

\begin{tabular}{|c|c|c|}
\hline No. & Identity & $\begin{array}{c}\text { Retention time } \\
\text { (minutes) }\end{array}$ \\
\hline 1 & Diphenoxylic acid & 12.1 \\
\hline 2 & Diphenoxylate Hydrochloride & 15.5 \\
\hline 3 & Nitrile amide & 28.5 \\
\hline 4 & Bromo diphenyl Butro nitrile & 32.4 \\
\hline
\end{tabular}

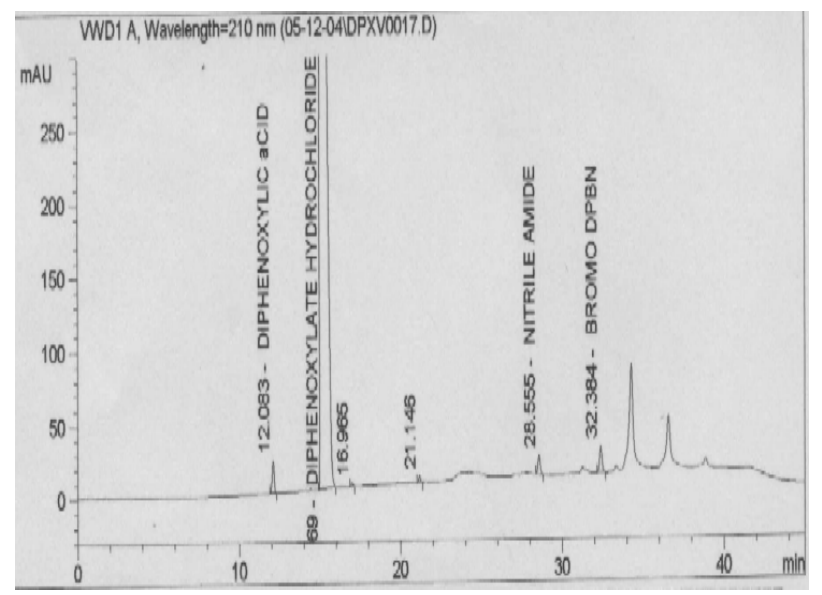

Figure 1. Typical chromatogram for Diphenoxylate hydrochloride spiked with $0.5 \%$ impurity level

\subsection{Parameters Performed for Analytical Validation}

\subsubsection{Suitability Tests}

System Suitability Solution was prepared as described under preparation of solutions and the solution was injected in replicate.

\subsubsection{Specificity Study}

To demonstrate specificity of the analytical method, Diphenoxylate hydrochloride and its impurities i.e. Diphenoxylic acid, Nitrileamide and Bromodiphenyl butronitrile were subjected to 'Forced degradation studies'. In 'Forced degradation studies, Diphenoxylate hydrochloride and its impurities were subjected to the following stress conditions

Given treatment with 1N Methanolic Hydrochloric acid , 1 $\mathrm{N}$ Methanolic Sodium hydroxide, 30\% v/v Hydrogen peroxide, Ultra-violet light (254 nm- photo degradation), Dry heat at $105^{\circ} \mathrm{C}$ (thermal degradation) and Heat \& humidity $\left(40{ }^{\circ} \mathrm{C}-75 \% \pm 5 \% \mathrm{RH}\right)$.

For acid, base and oxidation stress studies, the solutions of the Diphenoxylate hydrochloride, Nitrileamide and Bromo DPBN were prepared in Methanol. For acid, base and oxidation stress studies, the Diphenoxylate Acid was first dissolve in $1 \mathrm{~mL}$ Dimethylsulphoxide and then solution prepared in Methanol.

The concentration of the drug substance Diphenoxylate hydrochloride and the impurities was $0.3 \mathrm{mg} / \mathrm{mL}$. The exposure time was 3 days and 7 days.

For humidity, thermal and photo stability studies, known amount of Diphenoxylate hydrochloride and the impurities were exposed to heat, ultra violet light and humidity. The concentration of the drug substance Diphenoxylate hydrochloride and the impurities was $0.3 \mathrm{mg} / \mathrm{mL}$. The exposure time was 3 days.

\subsubsection{Detection Limit (DL) and Quantitation Limit (QL) Study}

A series of solutions were prepared by quantitative dilutions of the stock solution of Diphenoxylate hydrochloride, Diphenoxylic acid, Nitrileamide and Bromo DPBN working standard to obtain solutions in the range $2.0 \%$ to $10.0 \%$ of the working concentration solution (i.e. $0.1 \mathrm{ppm}$ to $0.5 \mathrm{ppm}$ ).

Each solution was injected in duplicate into the chromatograph and the peak response of each solution was recorded. The mean peak response for each concentration was calculated.

A graph of mean peak area vs. concentration (\%) was plotted and the equation of regression line and the residual standard deviation was determined. The calculations were done as follows:

Calculation:

$$
\mathrm{LOD}=\frac{3.3 \sigma}{\mathrm{-S}} \quad \mathrm{LOQ}=\frac{10 \sigma}{\mathrm{-a}} \mathrm{S}
$$

Where,

$\sigma=$ Residual Standard Deviation 


\section{$\mathrm{S}=$ Slope}

For QL the relative standard deviation for six replicate injections was less than $6.0 \%$.

\subsubsection{Linearity}

Linearity solutions were prepared by quantitative dilutions of the stock solution of Diphenoxylate hydrochloride, Diphenoxylic acid, Nitrileamide and Bromo DPBN working standard to obtain solutions in the range from the Quantitation Limit to $200 \%$ of the working concentration solution (i.e. $0.25 \mathrm{ppm}$ to $10.0 \mathrm{ppm}$ ). Each solution was injected into the chromatograph in duplicate and the mean peak areas were calculated.

A graph of mean peak area vs. concentration (\%) was plotted and the equation of regression line was determined. The slope, intercept and correlation coefficient of the regression line were calculated.

The graphs of linearity curve for Diphenoxylate hydrochloride and its impurity is given as follows;

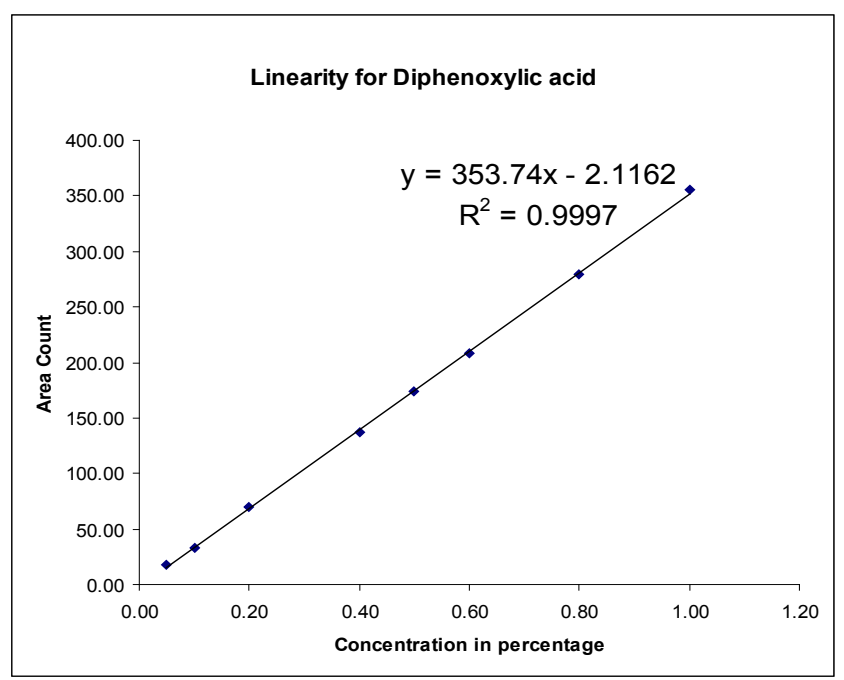

Figure 2. Linearity for Diphenoxylic acid

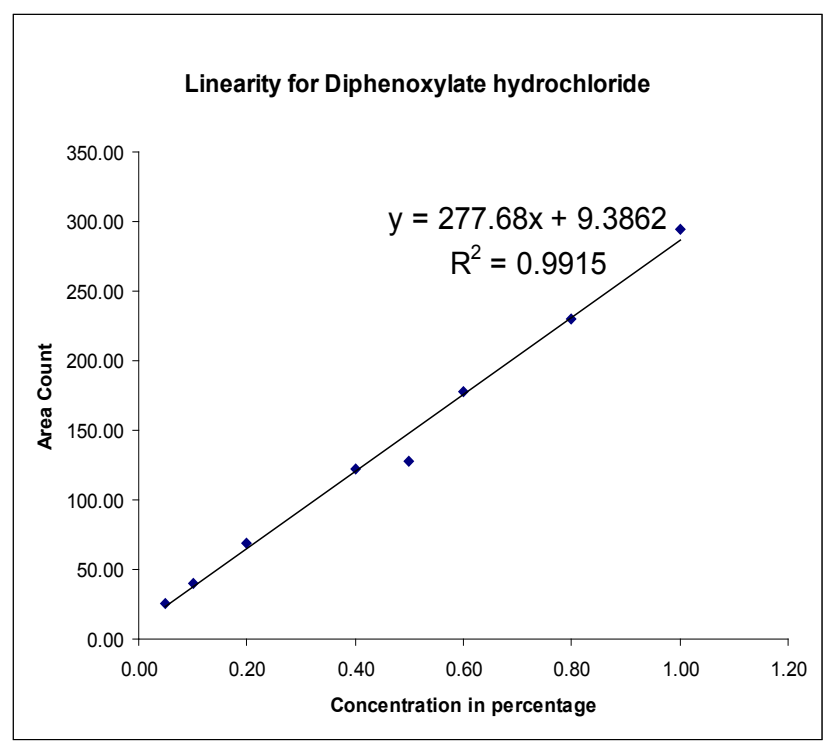

Figure 3. Linearity for Diphenoxylate hydrochloride

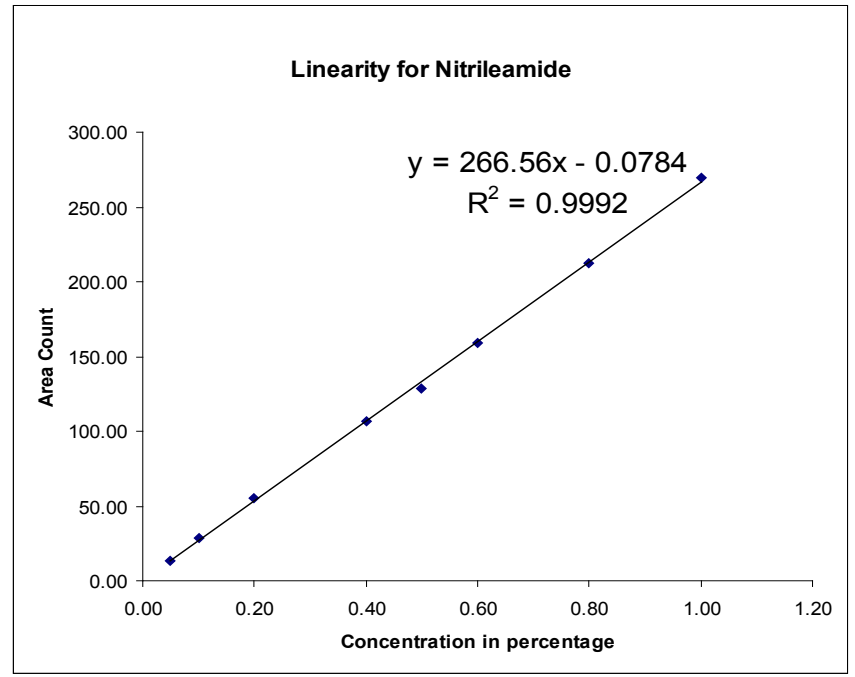

Figure 4. Linearity for Nitrile amide

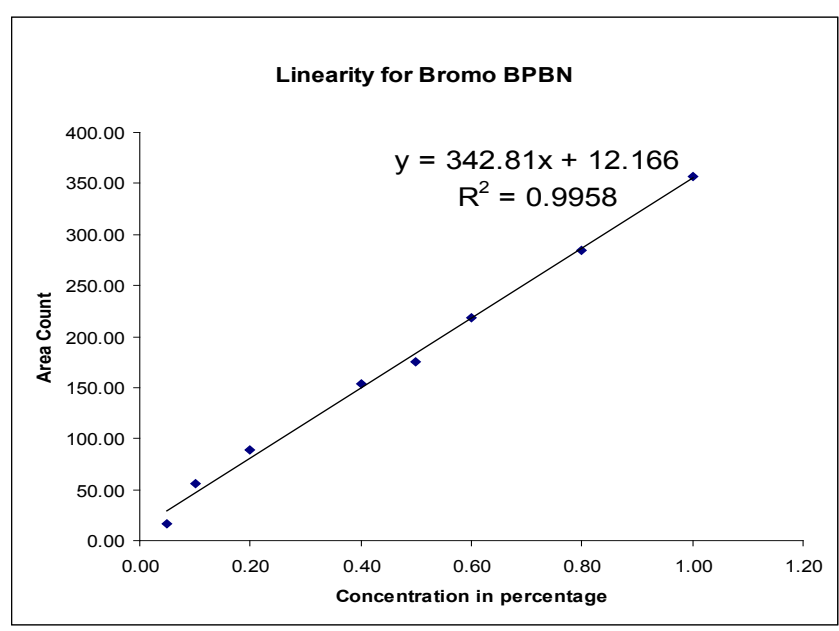

Figure 5. Linearity for Bromo DPBN

Table 3. Determination of Relative Retention factor (RRF) of Diphenoxylate hydrochloride and its impurities form the slope

\begin{tabular}{|c|c|c|c|c|}
\hline No. & Identity & $\begin{array}{c}\text { Slope from } \\
\text { Linearity } \\
\text { Experiment }\end{array}$ & (RRT) & (RRF) \\
\hline 1 & Diphenoxylic acid & 353.74 & 0.78 & 1.27 \\
\hline 2 & Diphenoxylate Hydrochloride & 277.68 & 1.00 & 1.00 \\
\hline 3 & Nitrileamide & 266.56 & 1.85 & 0.96 \\
\hline 4 & Bromo diphenyl Butro nitrile & 342.81 & 2.10 & 1.23 \\
\hline
\end{tabular}

\subsubsection{Precision}

\section{a) System Precision}

20ul of working concentration (i.e. $5 \mathrm{ppm}$ ) solution was injected in replicates. Mean peak area for Diphenoxylate hydrochloride, Diphenoxylic acid, Nitrileamide and Bromo DPBN were calculated.

\section{b) Repeatability}

Diphenoxylate hydrochloride sample was weighed in six different flasks. Each solution was analyzed against a freshly prepared standard of Diphenoxylic acid, Diphenoxylate hydrochloride, Nitrileamide and Bromo DPBN. The mean, standard deviation and relative standard deviation of the results for standard were calculated. 
Table 4. The results for Detection Limit (DL) and Quantitation Limit (QL)

\begin{tabular}{|c|c|c|c|}
\hline Components & Limit of Detection (\%) & Limit of Quantitation (\%) & RSD (\%) \\
\hline Diphenoxylate Acid & 0.001 (i.e.0.005ppm) & $0.05($ i.e. $0.25 \mathrm{ppm})$ & 0.75 \\
\hline Diphenoxylate hydrochloride & 0.009 (i.e.0.045ppm) & $0.05($ i.e. $0.25 \mathrm{ppm})$ & 2.47 \\
\hline Nitrileamide & $0.018($ i.e.0.090ppm) & $0.05($ i.e. $0.25 \mathrm{ppm})$ & 1.24 \\
\hline Bromo DPBN & $0.003($ i.e. $0.015 \mathrm{ppm})$ & $0.05($ i.e. $0.25 \mathrm{ppm})$ & 0.73 \\
\hline
\end{tabular}

c) Intermediate Precision

The repeatability experiment was repeated on different day and the results were compared with repeatability experiment.

\subsubsection{Accuracy}

Diphenoxylate hydrochloride sample was weighed in three different flasks. One of these flasks were spiked with solutions of Diphenoxylic acid, Nitrileamide and Bromo DPBN working standard so as to obtain solution at the concentration level of quantitation limit of Diphenoxylic acid, Diphenoxylate hydrochloride, Nitrileamide and Bromo DPBN. Similar solutions were prepared by spiking at $100 \%$ and $160 \%$ level of Diphenoxylic acid, Nitrileamide and Bromo DPBN of working concentration. Each level was analyzed against a freshly prepared standard of Diphenoxylic acid, Diphenoxylate hydrochloride, Nitrileamide and Bromo DPBN. The mean, standard deviation and relative standard deviation of the results were calculated.

\subsubsection{Range}

Range was defined once linearity, precision, and accuracy had been established. Based on linearity, precision and accuracy, it was determined that the range of the analytical method is from $0.05 \%$ to $0.8 \%$ of working concentration for all the three known impurities with respect to Diphenoxylate hydrochloride.

\subsubsection{Robustness}

The HPLC analysis was carried out using the method outlined in the Methodology section by spiking the sample of Diphenoxylate hydrochloride working standard with Diphenoxylate hydrochloride working standard, Diphenoxylate hydrochloride working standard, Diphenoxylate hydrochloride working standard and Diphenoxylate hydrochloride working standard solution at the working concentration level. The following alterations in the chromatographic conditions were then carried out:

a) Changing the wavelength of the detector $(210 \mathrm{~nm} \pm$ 2nm)

b) Changing flow rate $(2.2 \pm 0.2 \mathrm{~mL} / \mathrm{min})$

The difference between the results obtained in known impurities and unknown impurities in accordance with normal method and analysis by altered method were calculated and the system suitability test criteria were evaluated for every change.

\section{Result and Discussion}

\subsection{System Suitability}

Diphenoxylate hydrochloride and all the three impurities i.e. Diphenoxylic acid, Nitrile amide and Bromo DPBN were well resolved from each other.

\subsection{Specificity}

The method was found specific for Diphenoxylate hydrochloride, Diphenoxylic Acid, Nitrileamide and Bromo DPBN as there is no interference of degradants at the retention time of Diphenoxylate hydrochloride and the three known impurities as shown by the forced degradation studies under various stress conditions. Diphenoxylate hydrochloride and the three known impurities were found spectrally pure under the various stress condition.

For QL, the relative standard deviation for six replicate injections was less than $6.0 \%$ for Diphenoxylate hydrochloride and its impurities at $0.25 \mathrm{ppm}$.

\subsection{Linearity}

Correlation Coefficient for Diphenoxylate hydrochloride and all its impurities were more than 0.995 in the range of Limit of quantitation (i.e. $0.25 \mathrm{ppm}$ ) to $200 \%$ (i.e. $10.0 \mathrm{ppm}$ ).

\subsection{Precision}

\section{a) System precision}

The relative standard deviation of the results for System precision was less than $6.0 \%$ for Diphenoxylate hydrochloride and its impurity.

\section{b) Repeatability}

The relative standard deviation of the results for Repeatability was less than $6.0 \%$.

c) Intermediate precision

The relative standard deviation for System precision for Intermediate Precision was less than $6.0 \%$ for Diphenoxylate hydrochloride and its impurities.

\subsection{Accuracy}

The relative standard deviation of the results for System precision was less than $6.0 \%$ for Diphenoxylate hydrochloride and its impurity. The recovery at level of quantitation limit $(0.25 \mathrm{ppm})$ was within $70.0 \%$ to $130.0 \%$ for $\mathrm{Di}$ phenoxylic acid, Nitrile amide and Bromo DPBN. The individual recoveries at the $100 \%$ (i.e. spike at $5 \mathrm{ppm}$ ) and 
$160 \%$ level (spike at $8.0 \mathrm{ppm}$ ) were within $80.0 \%$ to $120.0 \%$ for Diphenoxylic acid, Nitrile amide and Bromo DPBN. The mean recovery was within $80.0 \%$ to $120.0 \%$ for $\mathrm{Di}$ phenoxylic acid, Nitrile amide and Bromo DPBN.

\subsection{Range}

Based on linearity, precision and accuracy, it was determined that the range of the analytical method is from $0.05 \%$ (i.e. $0.25 \mathrm{ppm}$ ) to $0.8 \%$ (i.e. $8.0 \mathrm{ppm}$ ) for all the three known impurities and unknown impurities with respect to Diphenoxylate hydrochloride.

\subsection{Robustness}

There was no significant difference in the results obtained by the normal method and those obtained by carrying out deliberate changes in the method.

\section{Conclusions}

The suggested method can be successfully used to estimate known and unknown impurities present in the Diphenoxylate hydrochloride bulk drug.

\section{ACKNOWLEDGEMENTS}

Author are thankful to M/S RPG LIFE Sciences Limited for supplying gift samples of drugs for carry out this work.

\section{REFERENCES}

[1] The Indian Pharmacopoeia Volume I published by the controller of publication, New Delhi. P 260-261 (1996).

[2] European Pharmacopeia, Third Edition published accordance with convention on Elaboration of European Pharmacopoiea. P755-756.(1997)

[3] I. Jane, A. Mckinnon and R. J. Flanagan, J. Chromatogr. 1985, 323(2), 191-225.

[4] I. M. Jalal, S. T. Sa'sa, A.H. Abusaleh and H. S.Khalil, Anal. Letl 1985, 18(b20) 2551-68.

[5] R. Gill and B. Law, J Chromatogr. 198634 185-202..

[6] Dennis W. Hill and Karen J.Langner, J Liq Chromatogr. 1987, 10(2-3) 377-409.

[7] Minsi Gao, Qinghua Zhu and Jun Chen, Zhongguo Yiyao Gongye Zazhi 1991, 22(10), 457-9.

[8] Gary J. Lehr, J. AOAC Int 1996, 79(6) 1288-1293. 